\title{
NO Candida auris: Nitric Oxide in Nanotherapeutics to Combat Emerging Fungal Pathogen Candida auris
}

\author{
Levi G. Cleare ${ }^{1,+}\left(\mathbb{D}\right.$, Kevin L. Li ${ }^{2,+}$, Waleed M. Abuzeid ${ }^{3}$, Parimala Nacharaju ${ }^{4}$, \\ Joel M. Friedman ${ }^{4}$ and Joshua D. Nosanchuk ${ }^{1, *(\mathbb{D})}$ \\ 1 Department of Medicine (Division of Infectious Diseases) and Department of Microbiology and \\ Immunology, Albert Einstein College of Medicine, Bronx, NY 10461, USA; levi.cleare@einsteinmed.org \\ 2 Department of Otorhinolaryngology-Head and Neck Surgery, Albert Einstein College of Medicine, \\ Bronx, NY 10461 USA; kevin.li@einsteinmed.org \\ 3 Department of Otolaryngology: Head and Neck Surgery, University of Washington, Seattle, WA 98195, USA; \\ wabuzeid@uw.edu \\ 4 Department of Physiology and Biophysics, Albert Einstein College of Medicine, Bronx, NY 10461, USA; \\ parimala.nacharaju@einsteinmed.org (P.N.); joel.friedman@einsteinmed.org (J.M.F.) \\ * Correspondence: josh.nosanchuk@einsteinmed.org \\ + These authors contributed equally to this work.
}

Received: 20 May 2020; Accepted: 10 June 2020; Published: 12 June 2020

check for updates

\begin{abstract}
Candida auris (C. auris) is an emerging pathogenic fungal species that is especially worrisome due to its high mortality rates and widespread antifungal resistance. Previous studies have demonstrated the efficacy of nitric oxide (NO) nanoparticles on Candida species, and, to our knowledge, this is the first study to investigate the antifungal effects of a NO-generating nanoparticle on C. auris. Six C. auris strains were incubated with a nanoparticle (NAC-SNO-np), which releases $N$-acetylcysteine $\mathrm{S}$-nitrosothiol (NAC-SNO) and $\mathrm{N}$-acetylcysteine (NAC), and generates $\mathrm{NO}$, through colony forming unit (CFU) assays, and confocal laser scanning microscopy. NAC-SNO-np effectively eradicates planktonic and biofilm $C$. auris. Across all six strains, $10 \mathrm{mg} / \mathrm{mL}$ NAC-SNO-np significantly reduced the number of CFUs $(p<0.05)$ and demonstrated a $>70 \%$ decrease in biofilm viability $(p<0.05)$. NAC-SNO-np effectively eradicates planktonic C. auris and significantly reduces $C$. auris biofilm formation. Hence, this novel NO-releasing nanoparticle shows promise as a future therapeutic.
\end{abstract}

Keywords: Candida auris; C. auris; nitric oxide; NAC; nanoparticles; confocal microscopy

\section{Introduction}

Candida auris (C. auris) is an emerging pathogenic fungal species first isolated in Japan in 2009 [1]. C. auris simultaneously emerged on three separate continents and is notable in that isolates from each continent are from different clades [2]. C. auris has now spread to all continents except for Antarctica [3-9]. C. auris is phylogenetically most closely related to C. haemulonii. This phylogenetic relatedness has made the actual global distribution unclear, in part due to the inability to properly identify C. auris using classic microbiological techniques, commonly misidentifying it as the closely related C. haemulonii [10-12]. While the exact global distribution remains unclear, it is apparent that C. auris is quickly becoming more prevalent.

C. auris can colonize multiple body areas, ranging from the ears and nares to the groin and rectum. It can be acquired from contact with patients harboring $C$. auris. Indeed, Horton et al. demonstrated recently that $C$. auris formed biofilms in both synthetic sweat media, mimicking the physiologic conditions of the axilla, and on porcine skin [13]. Much more worrisome is acquisition from contact with contaminated surfaces with $C$. auris biofilms having been shown to survive simulated 
desiccation [13]. Reports of $C$. auris outbreaks in the United States show that these infections likely began in health care facilities and were transmitted nosocomially $[14,15]$. This is particularly concerning as $C$. auris harbors a predilection for causing severe candidemia in critically ill patients $[6,14,16,17]$. C. auris has an unclear mortality rate, with wide variation based on region and underlying comorbidities. In the United States, 30 day mortality rates related to candidemia exceed 50\% [14,16,18].

C. auris exhibits widespread resistance to antifungal agents. Lockhart et al. examined resistance rates in isolates from 54 patients representing the different clades and found that $93 \%$ of isolates were resistant to fluconazole, 35\% to amphotericin B, and 7\% to echinocandins [2]. This resistance profile has prompted physicians to prescribe echinocandins empirically for suspected C. auris infections [19]. It is further notable that $41 \%$ of isolates were resistant to two separate antifungal classes and $4 \%$ were resistant to all three antifungal classes [2,20]. In light of the widespread antifungal resistance and significant morbidity and mortality rates, it is imperative to develop novel approaches for combatting C. auris.

Nitric oxide (NO) is an endogenously generated diatomic gas that is important for cellular signaling, vascular modulation, and immune function, and is a vital component of mammalian host defenses [21]. NO is an innate immune system product and acts as both a cytostatic and cytotoxic broad-spectrum antimicrobial agent. NO has antimicrobial properties against many bacteria, yeasts, helminths, protozoa, and viruses [22]. Notably, in terms of fungi, NO has previously demonstrated antimicrobial efficacy against C. albicans [22-24]. Moreover, we have developed a NO nanoparticle delivery platform that has demonstrated antimicrobial efficacy against $C$. albicans through disruption of fungal growth and morphogenesis [25-27]. The NO nanoparticles can kill both hyphae and yeast forms within mature biofilms, predominantly by inducing apoptosis and subsequent cell necrosis [25]. Recently, Vargas-Cruz et al. demonstrated the efficacy of a nitroglycerin-citrate-ethanol catheter lock solution, which converts nitroglycerin into NO, in the eradication of $C$. auris biofilms in central line lumens. The authors further showed that the biofilm eradication was superior to common antifungal medications such as amphotericin B, fluconazole, and caspofungin, among others, in vitro [28].

In this study, we utilized a novel nanoparticle (NAC-SNO-np), which releases $N$-acetylcysteine $S$-nitrosothiol (NAC-SNO) to induce a sustained release of NO and explored the efficacy of NAC-SNO-np in disrupting $C$. auris. To our knowledge, this is the first study to investigate the antifungal effects of $\mathrm{NO}$ on both planktonic and biofilm forms of $C$. auris.

\section{Materials and Methods}

\subsection{Candida auris Strains}

A total of six C. auris strains were used in this study. Two clinical strains (MMC 1 and MMC 2) were isolated at the Montefiore Medical Center (MMC; Bronx, NY, USA) and we have previously characterized their resistance phenotypes and multi-omics signatures [29]. The samples were obtained with the written consent of all patients under a protocol that was approved by the institutional review boards (IRB Number: 2016-7455 approved on 2 July 2017) of the Albert Einstein College of Medicine and MMC. Four reference strains (CDC 0381, CDC 0383, CDC 0385, and CDC 0388), from major clades around the world, were obtained from the $C$. auris panel of the Centers for Disease Control (CDC; Atlanta, GA, USA) and Food and Drug Administration (FDA; Silver Spring, MD, USA) Antibiotic Resistance Isolate Bank (https://www.cdc.gov/drugresistance/resistance-bank/). Minimal Inhibitory Concentrations (MICs) for C. auris to amphotericin B, fluconazole and echinocandins from MMC [29], and the CDC have been previously described. Hence, the strains selected represent both susceptible and resistant isolates. All strains were stored in liquid nitrogen and, suspended in Sabouraud (SAB) broth with $50 \%$ glycerol, until use. Strains were grown in SAB broth for $24 \mathrm{~h}$ at $37^{\circ} \mathrm{C}$ using a rotary shaker set at $150 \mathrm{rpm}$. 


\subsection{C. auris Resistance Profiles}

Six diverse $C$. auris strains, clinical and reference, were used to examine the generalizability of the efficacy of the NAC-SNO-np against this species. These strains included the original Japanese strain and encompass four separate continents. Three of our six strains were resistant to fluconazole, and one strain (CDC 0383) was resistant to both fluconazole and caspofungin. No strain in our study was resistant to amphotericin B (Table 1).

Table 1. C. auris strains were collected from Montefiore Medical Center (MMC) and the Centers for Disease Control (CDC). Tentative MIC breakpoints (bolded values) for C. auris are reported from the CDC as: amphotericin $\mathrm{B} \geq 2$; caspofungin $\geq 2$; fluconazole $\geq 32$.

\begin{tabular}{ccccc}
\hline \multirow{2}{*}{ Fungal Isolate } & \multirow{2}{*}{ Origin } & \multicolumn{3}{c}{ MIC $(\mu \mathrm{\mu g} / \mathrm{mL})$} \\
\cline { 3 - 5 } & & Amphotericin B & Caspofungin & Fluconazole \\
\hline MMC 1 & Bronx, NY & 1.6 & 2 & $>256^{\text {a }}$ \\
\hline MMC 2 & Bronx, NY & 0.8 & 1.6 & 8 \\
\hline CDC 0381 & Japan & 0.38 & 0.125 & 4 \\
\hline CDC 0383 & South Africa & 0.38 & $\mathbf{1 6}$ & $\mathbf{1 2 8}$ \\
\hline CDC 0385 & Venezuela & 0.5 & 0.5 & $>\mathbf{2 5 6}$ \\
\hline CDC 0388 & Pakistan & 1.5 & 0.5 & $\mathbf{2 5 6}$ \\
\hline \multicolumn{2}{r}{${ }^{\text {a }}$ MMC1 was resistant to fluconazole at a concentration of $1000 \mu \mathrm{g} / \mathrm{mL}}$. \\
\end{tabular}

\subsection{Synthesis of NAC-SNO-np}

\subsubsection{Preparation of Sol-Gel}

The sol-gel was prepared with tetramethylorthosilicate (TMOS), as previously described [30,31]. Briefly, $3 \mathrm{~mL}$ of TMOS was hydrolyzed by $0.37 \mathrm{~mL}$ of $40 \mathrm{mM} \mathrm{HCl}$ in the presence of $2.5 \mathrm{~mL} \mathrm{MeOH}$ at $60^{\circ} \mathrm{C}$ for $1.5 \mathrm{~h}$. This mixture was diluted with $200 \mu \mathrm{L}$ of water and $1 \mathrm{~mL}$ of similarly hydrolyzed 3-aminopropyltrimethoxysilane (APTS). APTS introduces amino groups into the matrix and promoted sustained release of the enclosed contents in our previous studies [30]. The polymerization of this hydrolyzed TMOS was carried out at $40^{\circ} \mathrm{C}$. It formed a clear gel within $30 \mathrm{~min}$.

\subsubsection{Preparation of NAC-SNO-np}

Two grams of gel was mashed with a glass rod and cooled on ice. To this, $2 \mathrm{~mL}$ of freshly prepared, cold NAC-SNO $(900 \mu \mathrm{mol})$ was added, which was prepared by mixing $900 \mu \mathrm{mol}$ of nitrite and $1080 \mu \mathrm{mol}$ of NAC. The excess NAC ensured the complete conversion of nitrite into SNO. This leaves an unreacted NAC of approximately $180 \mu \mathrm{mol}$ in the mixture. The mixture was mixed on a lab rotator for $3 \mathrm{~h}$ at $4{ }^{\circ} \mathrm{C}$. The mixture was then centrifuged at $1900 \times g$ for $5 \mathrm{~min}$, supernatant separated, and particles lyophilized. The dried powder was ground into finer particles with a mortar and pestle (NAC-SNO-np). The NAC-SNO-nps were stored in aliquots at $-80^{\circ} \mathrm{C}$. The final concentration of NAC-SNO in nanoparticles was $1.2 \mu \mathrm{mol} / \mathrm{mg}$. The concentration of NAC in the particles was not determined. The particle size ranged from 200 to $2000 \mathrm{~nm}$, as determined by dynamic light scattering.

\subsection{Measuring the Release of NAC-SNO from NAC-SNO-np}

NAC-SNO-nps were dispersed in phosphate-buffered saline (PBS) at $5 \mathrm{mg} / \mathrm{mL}$ and mixed on a lab rotator at room temperature. Aliquots were drawn at specific time intervals, diluted 10 times, and centrifuged at $17,000 \times g$ at $4{ }^{\circ} \mathrm{C}$. The supernatants were separated and the absorbance at $335 \mathrm{~nm}$ was recorded on a spectrophotometer (Thermo Fisher Scientific, Waltham, MA, USA). Since S-nitrosothiols are temperature sensitive [32], the rate of decay of NAC-SNO at room temperature was also studied. 
NAC-SNO at comparable concentration to that in NAC-SNO-np was incubated in PBS at room temperature. Aliquots were diluted at specific time intervals and the absorbance at $335 \mathrm{~nm}$ was recorded.

\subsection{Measuring the Release of NO from NAC-SNO-np}

NO release from NAC-SNO-np and NAC-SNO was measured using a NO analyzer (Sievers 280i; GE Instruments, Boulder, CO, USA). NAC-SNO-np was dispersed in $5 \mathrm{~mL}$ of PBS at $5 \mathrm{mg} / \mathrm{mL}$ at room temperature. NAC-SNO at matching concentrations with NAC-SNO-np was used as a control. Release kinetics were analyzed using Sievers NO Analysis software (GE Instruments, Boulder, CO, USA) with 12 measurements per minute recorded for $280 \mathrm{~min}$.

\subsection{Planktonic and Biofilm Plate Preparation}

In order to prepare C. auris for plating, logarithmically growing fungal cells prepared the previous night were collected by centrifugation, washed twice with PBS, counted using a hemocytometer, and suspended in a chemically defined minimal medium $(20 \mathrm{mg} / \mathrm{mL}$ of thiamine, $30 \mathrm{mM}$ glucose, $26 \mathrm{mM}$ glycine, $20 \mathrm{mM} \mathrm{MgSO} 4.7 \mathrm{H} 2 \mathrm{O}$, and $58.8 \mathrm{mM} \mathrm{KH2PO} 4)$ supplemented with $5 \%$ fetal bovine serum (FBS; Atlanta Biologicals, Minneapolis, MN, USA) [25]. Two separate suspensions of C. auris were prepared, planktonic and biofilm. Planktonic cells were aspirated into $2 \mathrm{~mL}$ microcentifuge tubes where $100 \mu \mathrm{L}$ of a $2 \times 10^{7}$ cells/mL of fungal cell suspension were added to each tube. The planktonic tubes were then co-incubated with NAC-SNO-nps in a microtube rotator at $37^{\circ} \mathrm{C}$ for $24 \mathrm{~h}$. Biofilm preparations were plated onto a 96-well tissue culture treated microplate. The biofilm preparation was plated at a concentration of $1 \times 10^{7}$ cells $/ \mathrm{mL}$, with $200 \mu \mathrm{L}$ of fungal cell suspension in each well. The biofilm plate was incubated for $24 \mathrm{~h}$ to allow for mature biofilm formation prior to use in therapeutic testing.

\subsection{Determining Susceptibility of C. auris to NAC-SNO-np}

Optimal NAC-SNO-np dosage was determined by fungal cell growth curves performed on planktonic MMC 1 and MMC 2. Planktonic MMC strains were plated, as described above, at a concentration of $1 \times 10^{7}$ cells/mL on a 96-well microplate and incubated with an additional $100 \mu \mathrm{L}$ of increasing concentrations of NAC-SNO-np $(1.25,2.5,5$, and $10 \mathrm{mg} / \mathrm{mL})$ suspended in control solution for $48 \mathrm{~h}$. The optical density (OD) was measured at $492 \mathrm{~nm}$ in $24 \mathrm{~h}$ intervals.

\subsection{Measuring Planktonic and Biofilm Viability by CFU Killing Assay}

Colony forming unit (CFU) killing assays were used to determine the susceptibility of C. auris strains to NAC-SNO-np. Three treatment solutions were used in this study: NAC-SNO-np, blank-np, and a control solution consisting of Minimal medium with 5\% FBS. Blank-np consisted of the nanoparticle scaffolding without the addition of NAC or NAC-SNO. The 6 C. auris strains were grown as planktonic and biofilm forms and tested with these three solutions. For each experiment, treatment conditions were tested in triplicate. Each experiment was repeated in triplicate for every strain of $C$. auris, totaling 9 data points for each treatment group.

Planktonic C. auris was grown, as described above, and $100 \mu \mathrm{L}$ of fungal cell suspensions were incubated with a $100 \mu \mathrm{L}$ solution of either $10 \mathrm{mg} / \mathrm{mL}$ NAC-SNO-np, $10 \mathrm{mg} / \mathrm{mL}$ blank-np, or control solution for $24 \mathrm{~h}$. SAB agar plates were inoculated with $100 \mu \mathrm{L}$ of a $10^{6}$ to $10^{8}$ times diluted solution from each treatment group and incubated for $48 \mathrm{~h}$ before CFUs were counted. Biofilm C. auris was grown as described above in 96 well plates. After $24 \mathrm{~h}$ of initial growth, the liquid medium was gently removed by pipette and $200 \mu \mathrm{L}$ of a $10 \mathrm{mg} / \mathrm{mL}$ solution of NAC-SNO-np, $10 \mathrm{mg} / \mathrm{mL}$ blank-np, or a control solution were added followed by another $24 \mathrm{~h}$ incubation. After the incubation period, the fungal cells were collected by vigorous scraping and pipetting, and $100 \mu \mathrm{L}$ of a $10^{6}$ to $10^{8}$ times diluted solution from each treatment group was then plated onto SAB agar plates, incubated for $48 \mathrm{~h}$, and CFUs were counted. 


\subsection{Evaluating NAC-SNO-np on Biofilm Viability by Confocal Laser Scanning Microscopy}

MMC 1 and MMC 2 biofilms were grown, as described above, except that $500 \mu \mathrm{L}$ of a fungal cell suspension was inoculated into each chamber of an 8 well LabTek II Chambered \#1.5 Coverglass System (Thermo Fisher Scientific, Waltham, MA, USA). Biofilms were incubated at $37^{\circ} \mathrm{C}$ for $48 \mathrm{~h}$, and the medium was replaced after $24 \mathrm{~h}$. Both fungal strains were treated with a $10 \mathrm{mg} / \mathrm{mL}$ NAC-SNO-np treatment solution for $24 \mathrm{~h}$. After treatment, biofilms were stained with LIVE/DEAD FungaLight (Thermo Fisher Scientific, Waltham, MA, USA). Images of biofilms were obtained using a TCS SP5 Confocal Laser Scanning Microscope (Leica Microsystems, Wetzlar, Germany) configured to a $405 \mathrm{~nm}$ diode and $488 \mathrm{~nm}$ argon laser with a $63 \times$ objective. Five Z-stacks of each individual sample were taken corresponding to the areas of highest biofilm growth. Each Z-stack consisted of $0.75 \mu \mathrm{m}$ individually sliced images of the same area of biofilm. Volocity software (Version 6.5.1, PerkinElmer, Waltham, MA, USA) was used to quantify biofilm viability and biomass within each Z-stack. This experiment was performed in triplicate for both strains.

\subsection{Evaluating Blank-np Penetration by Confocal Microscopy}

To evaluate the ability of blank-np to penetrate through C. auris biofilms, MMC 1 and MMC 2 biofilms were grown in an 8 well chamber, as described above. After $48 \mathrm{~h}$ of growth, each biofilm was stained with calcofluor white stain to visualize the fungal cell wall (Eng. Scientific, Inc. Clifton, NJ, USA) and treated with $200 \mu \mathrm{L}$ of a $1 \mathrm{mg} / \mathrm{mL}$ Syto-9-stained blank-np solution for $24 \mathrm{~h}$. Biofilms were then imaged using a TCS SP5 Confocal Laser Scanning Microscope with the same configurations as above. Three Z-stacks ( $0.75 \mu \mathrm{m}$ individual sliced images) were taken from each well. Volocity software was used to evaluate Blank-np biofilm penetration.

\subsection{Statistical Analysis}

Statistical analysis was performed using GraphPad Prism (Version 8.3, GraphPad Software, La Jolla, San Diego, CA, USA). One-way ANOVA, and independent t-tests were used to analyze differences between CFU treatment groups and biofilm volume, respectively. Statistical significance was set at the standard $p<0.05$.

\section{Results}

\subsection{NAC-SNO-np NAC-SNO Release Curve}

The release rate of NAC-SNO from NAC-SNO-np was studied at room temperature. Only $50 \%$ of the total amount of enclosed NAC-SNO released in the first $4 \mathrm{~h}$. The maximum amount released in these $4 \mathrm{~h}$ is taken as the total amount to calculate the cumulative release, as the decay of NAC-SNO disrupted further measurements. As can be seen in Figure 1, more than $90 \%$ of NAC-SNO was released instantaneously followed by a very slow release. The initial rapid release may be due to the release from the surface of the nanoparticles, and the contents contained within the particles are released at a slower rate. The decline in the curve with time is an indication of dissociation of NAC-SNO at room temperature. This decline is more prominent at later time points, due to which the released amounts are underestimated. 


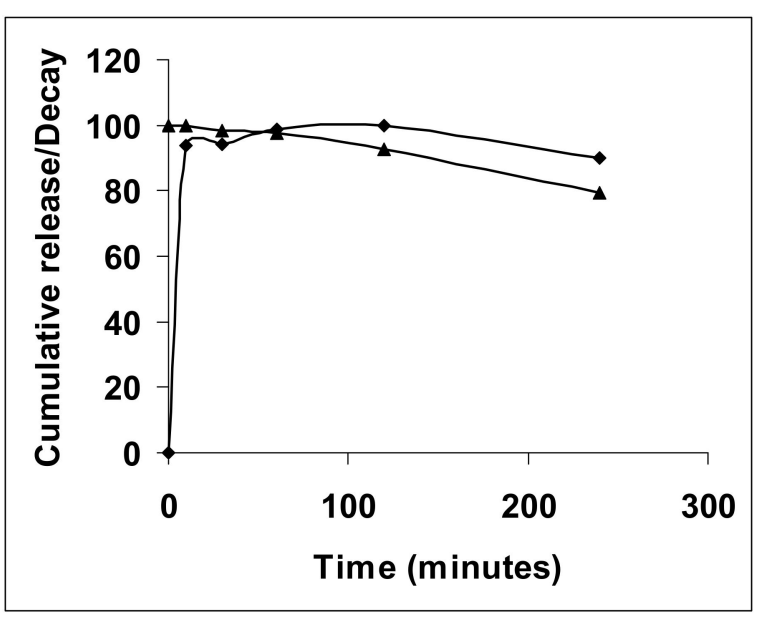

Figure 1. Rate of NAC-SNO release from nanoparticles; NAC-SNO-nps were dispersed in PBS, pH 7.4 at room temperature. The amount of NAC-SNO released (diamond) was determined from the absorbance of the buffer at $335 \mathrm{~nm}$. The rate of decay of NAC-SNO (triangle) at room temperature is also shown.

\subsection{NAC-SNO-np NO Release Curve}

The rate of NO release from NAC-SNO-np in PBS, $\mathrm{pH} 7.4$, was studied at room temperature using a NO analyzer (Figure 2). NAC-SNO-np initially released NO approximately $4 \mathrm{ppm}$ and exhibited a gradual decrease in rate to $1.5 \mathrm{ppm}$ by $280 \mathrm{~min}$. This profile matches the rate of NAC-SNO release from the particles-A rapid release followed by a steady, slow release (Figure 1). NAC-SNO released an initial bolus of $\mathrm{NO}$ (approximately $4.5 \mathrm{ppm}$ ) and dropped to $1.3 \mathrm{ppm}$ within $5 \mathrm{~min}$. After, a slow increase followed that equilibrated at a steady release rate of $1.7 \mathrm{ppm}$, which was observed until the end of the experiment 280 min later.

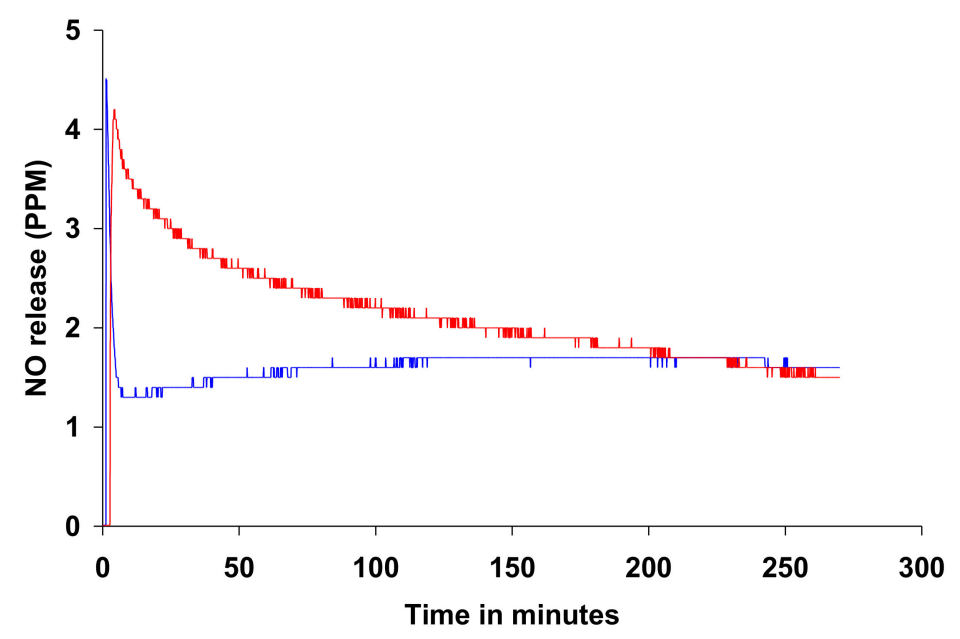

Figure 2. Rate of nitric oxide release from NAC-SNO-np, as monitored by a NO analyzer; NAC-SNO-np (red) or NAC-SNO (blue) was added to PBS, pH 7.4, at room temperature.

\subsection{Spectrophotometric Assay}

Spectrophotometric assay of MMC 1 and MMC 2 treated with NAC-SNO-np demonstrated complete inhibition of fungal cell growth under planktonic conditions for both strains at a final NAC-SNO-np concentration of $10 \mathrm{mg} / \mathrm{mL}$ (Figure 3). Dose-dependent growth curves were performed in triplicate. NAC-SNO-np decreased $C$. auris growth in a dose-dependent manner, with a final concentration of $10 \mathrm{mg} / \mathrm{mL}$ NAC-SNO-np sufficient to arrest planktonic C. auris. As a result, $10 \mathrm{mg} / \mathrm{mL}$ was chosen as the appropriate NAC-SNO-np concentration for the subsequent experiments in this study. 
C. auris MMC 1

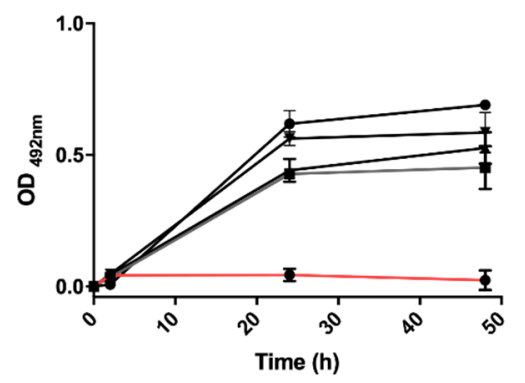

C. auris MMC 2

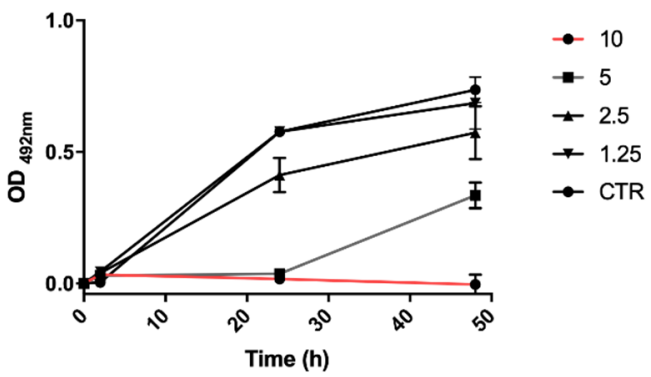

Figure 3. Dose-dependent growth curves were generated for planktonic C. auris MMC 1 and MMC 2 to determine the minimum concentration of NAC-SNO-np to eradicate fungal cells.

\subsection{CFU Killing Assay}

Planktonic and biofilm CFU killing assays were performed for the six strains of C. auris (Figure 4). Varying rates of colony formation were seen between each strain of C. auris, with the CDC strains forming many more colonies than their MMC counterparts. With the exception of the biofilm preparation of MMC 2, each strain treated with NAC-SNO-np demonstrated a significant reduction for both planktonic and biofilm C. auris compared to control conditions $(p<0.05)$. Specifically, planktonic MMC 1 had a $9.59 \log$ decrease and biofilm MMC 1 had a 9.38 log decrease. Planktonic and biofilm CDC 0381 had a 10.2 and 9.68 log reduction in CFUs, respectively, while planktonic and biofilm CDC 0383 CFUs had a $9.88 \mathrm{log}$ reduction and a $2.65 \mathrm{log}$ reduction, respectively. Planktonic CDC 0385 had a $3.13 \mathrm{log}$ reduction and the biofilm CFUs had a $2.21 \mathrm{log}$ reduction. Finally, planktonic and biofilm CDC 0388 had a 1.49 and $3.04 \log$ reduction, respectively. MMC 2 planktonic plates had a 9.18 log reduction, and biofilm plates had a $0.98 \mathrm{log}$ reduction, remaining statistically significant $(p<0.01)$. Across the six strains, there was a non-significant reduction in planktonic CFU count between the control group and the blank-np treatment group, except for CDC 0385, where the planktonic colonies demonstrated a significant decrease in fungal growth due to blank-np $(p<0.001)$. Furthermore, for the biofilm colonies, MMC 1 and CDC 0381 showed a significant decrease in CFUs $(p<0.05)$.
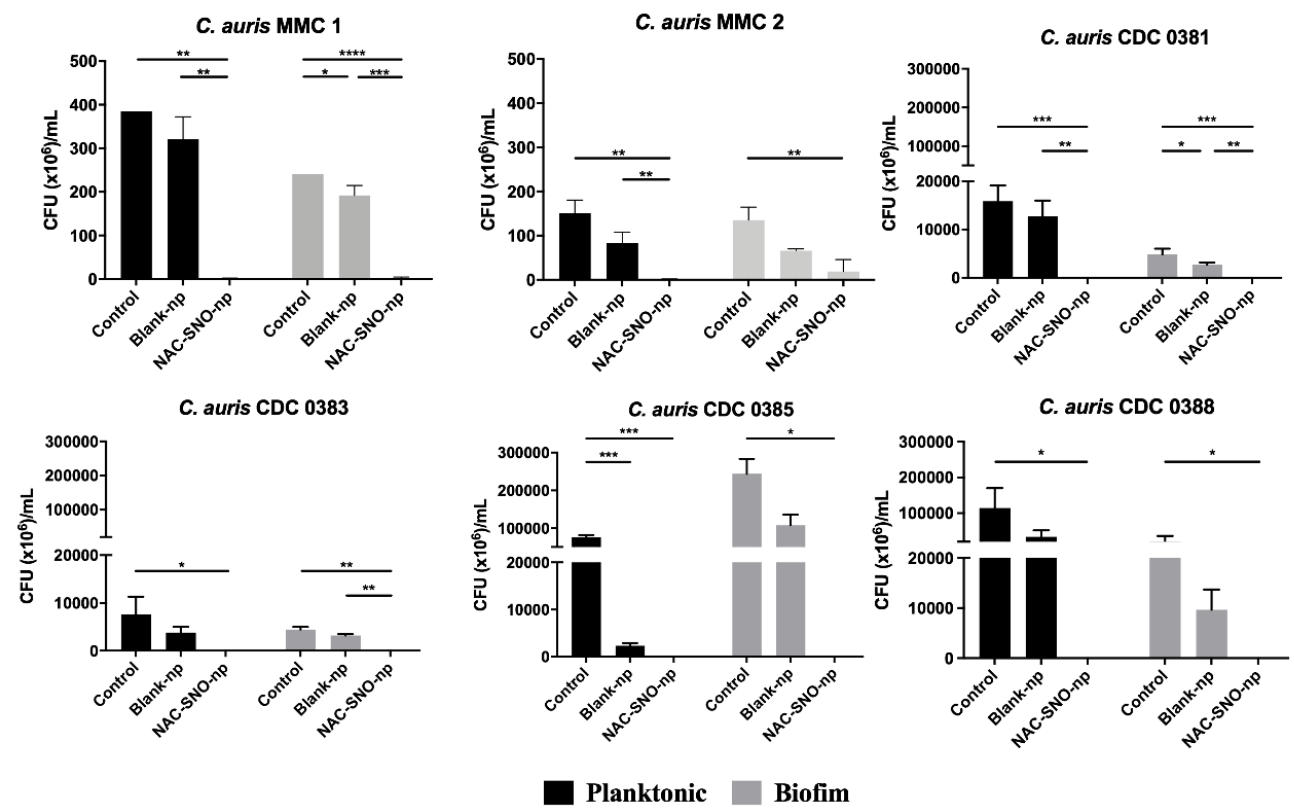

Figure 4. Planktonic and biofilm C. auris were incubated for $48 \mathrm{~h}$ and treated with NAC-SNO-np $(10 \mathrm{mg} / \mathrm{mL})$. CFUs were counted after $24 \mathrm{~h}$. Statistical analysis was performed by one-way Anova with Tukey's multiple comparison test. ${ }^{*} p<0.05,{ }^{* *} p<0.01,{ }^{* * *} p<0.001,{ }^{* * * *} p<0.0001$. 


\subsection{Biofilm Viability by Confocal Microscopy}

For both MMC 1 and MMC 2, control wells had 80 and 90\% viability, respectively, as measured by confocal microscopy biofilm volume and viability staining (Figures 5 and 6). After a $24 \mathrm{~h}$ treatment with a $10 \mathrm{mg} / \mathrm{mL}$ NAC-SNO-np solution, both strains demonstrated significant susceptibility to the NAC-SNO-np treatment. MMC 1 showed a $74 \%$ reduction in biofilm viability, which corresponds to a statistically significant $0.58 \log$ reduction $(p<0.05$; Figure 6). Compared to MMC 1 , the MMC 2 biofilm was slightly less susceptible to NAC-SNO-np treatment, but still demonstrated a statistically significant $71 \%$ decrease or $0.54 \log$ reduction in biofilm viability $(p<0.01)$.
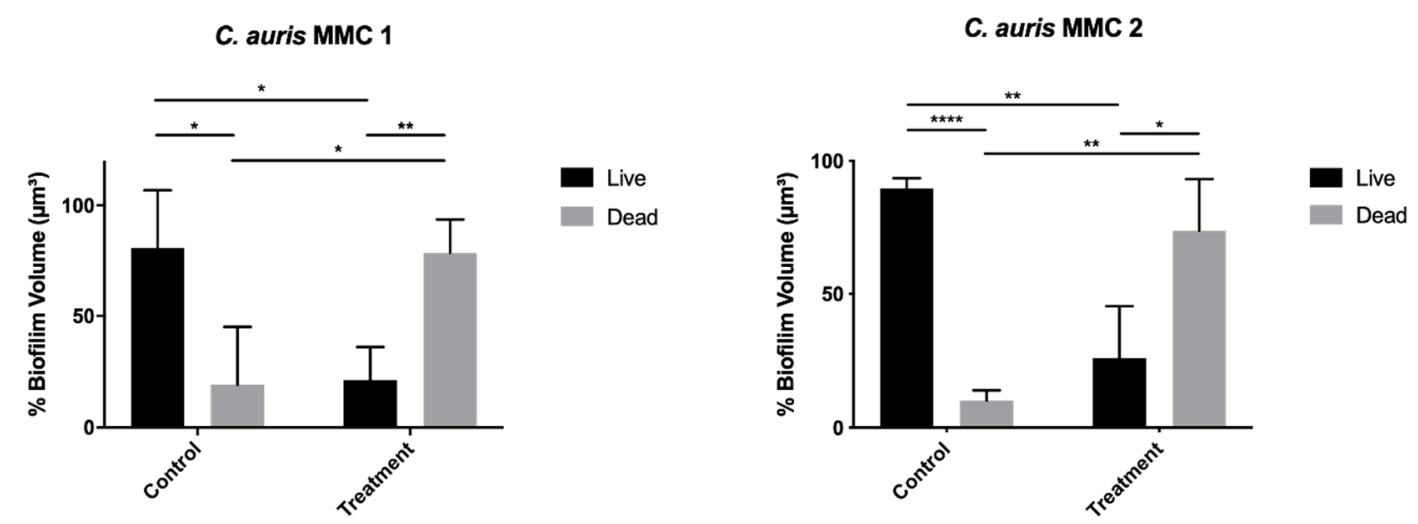

Figure 5. C. auris biofilms were grown for $48 \mathrm{~h}$ and treated with $500 \mu \mathrm{L}$ of NAC-SNO-np $(10 \mathrm{mg} / \mathrm{mL})$ for $24 \mathrm{~h}$. Graphs represent averages and standard deviations. Statistical analysis was performed by independent t-tests. ${ }^{*} p<0.05,{ }^{* *} p<0.01$, and ${ }^{* * *} p<0.0001$.

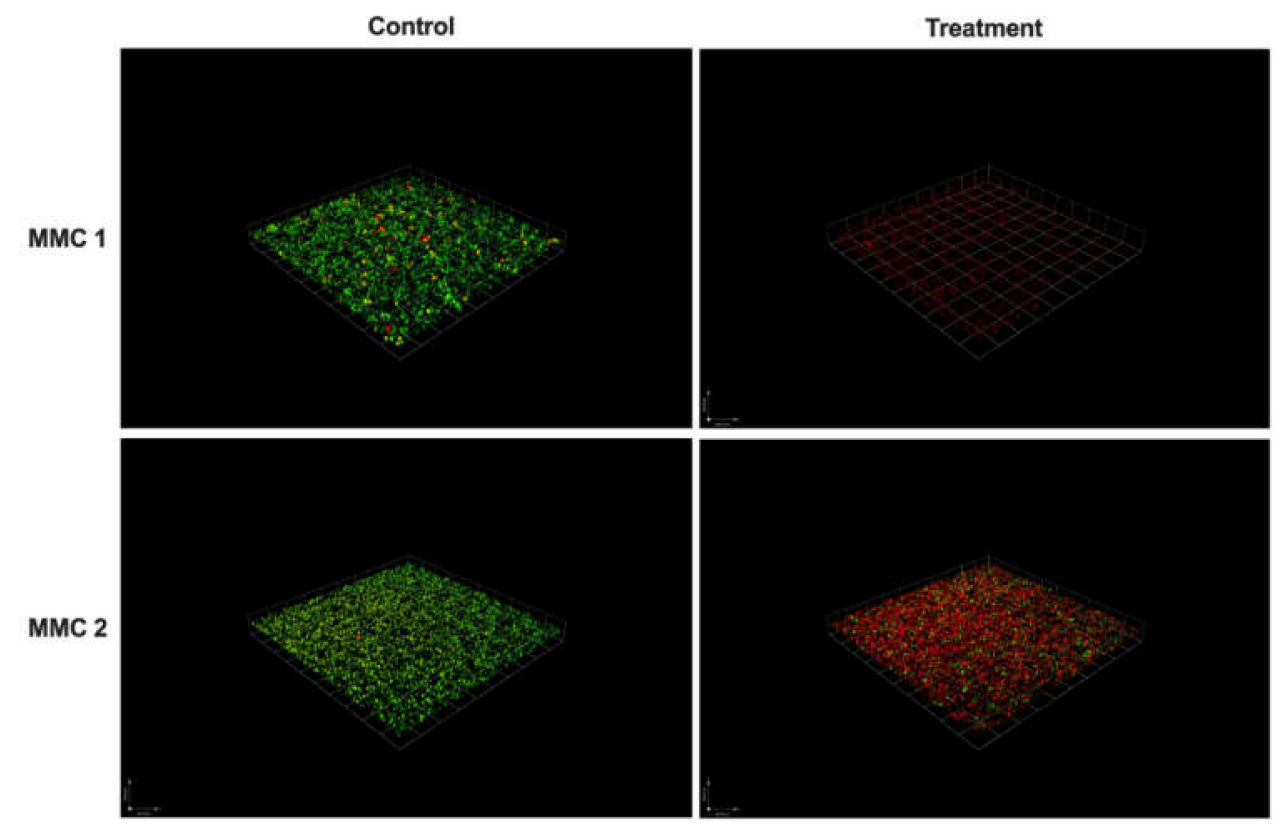

Figure 6. C. auris biofilms grown for $48 \mathrm{~h}$ and treated with $500 \mu \mathrm{L}$ of NAC-SNO-np $(10 \mathrm{mg} / \mathrm{mL})$ for 24 h. C. auris biofilm stained with FungaLight (live: green; dead: red). Scale bar represents $100 \mu \mathrm{m}$.

\subsection{Blank-np Biofilm Penetration}

Confocal microscopy imaging of MMC 1 and MMC 2 biofilms visually shows homogenous distribution of the particles within the wells with little clumping and demonstrates effective penetration of our nanoparticles (Figure 7). 


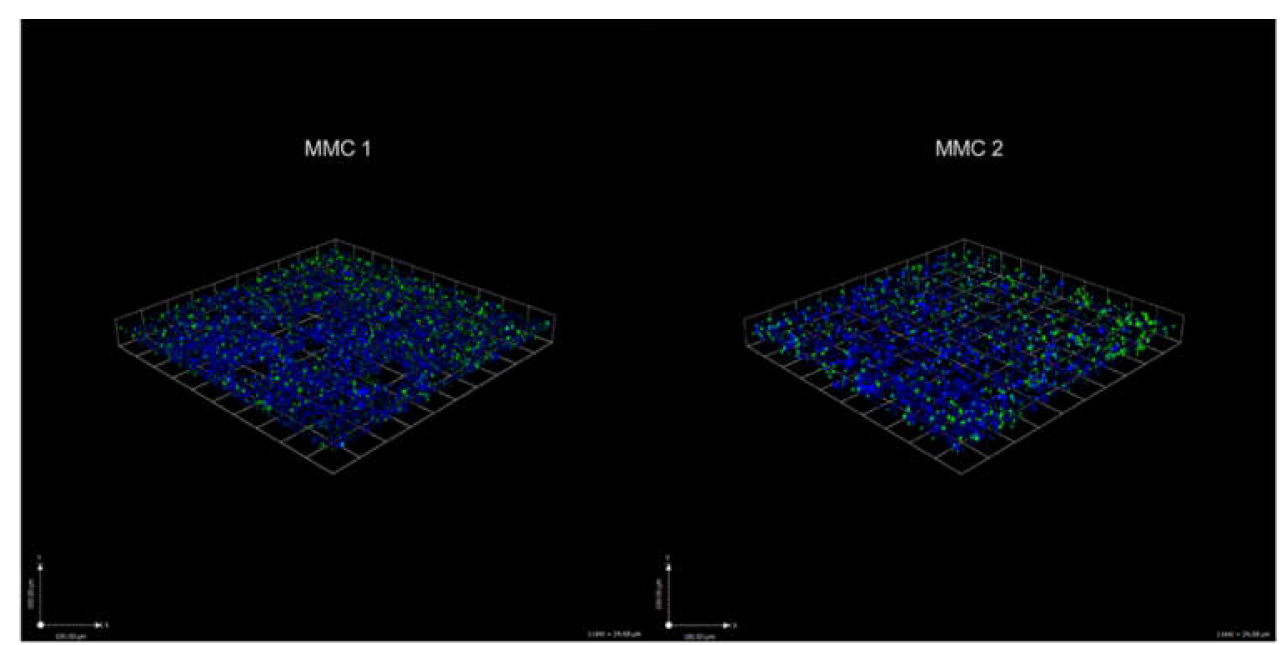

Figure 7. C. auris biofilms grown for $48 \mathrm{~h}$ and treated with $200 \mu \mathrm{L}$ of blank-np $(1 \mathrm{mg} / \mathrm{mL})$ for $24 \mathrm{~h}$. C. auris biofilm stained with calcoflour (cell wall: blue) and Syto-9 (blank-np: green). Scale bar represents $100 \mu \mathrm{m}$.

\section{Discussion}

Biofilms are a principal form of microbial growth [33]. They are critical to the development of certain infections, especially in the presence of foreign material such as indwelling catheters. Fungal cells in biofilms are more resistant to antifungal drugs than planktonic cells. Biofilms are complex surface-associated cell populations embedded in an extracellular matrix (ECM) that possess distinct phenotypes compared to their planktonic cell counterparts [33]. Other contributing factors include metabolic heterogeneity intrinsic to biofilms, biofilm-associated upregulation of efflux pump genes, nutrients, quorum-sensing molecules, and surface contact. The actual fold increase in resistance varies with both the drug and species. Candida albicans and Candida parapsilosis biofilms are relatively resistant to fluconazole, amphotericin B, nystatin, voriconazole, and others [33], and it appears that C. auris has many of the same mechanisms, particularly the efflux pump, that contribute to the notorious recalcitrance of its biofilm to common antifungal medications [34].

C. auris is an emerging fungal pathogen that has garnered attention due to its widespread antifungal resistance and high mortality rates. In the present work, we demonstrate the efficacy of a novel NAC-SNO-np treatment platform against six strains of both planktonic and biofilm forms of $C$. auris. This nanoparticle formulation releases NO, NAC, and NAC-SNO-All of which have previously demonstrated antimicrobial effects [35-38]. Thiol groups from NAC have been shown to exhibit antimicrobial activity through the formation of peroxynitrite [39]. The antibacterial activity of S-nitrosothiols is attributed to their capability of modifying thiols on enzymes by transnitrosation [40]. Figure 2 portrays the similar initial peak of NO release in both the NAC-SNO-np and NAC-SNO solution. Thereafter, we demonstrate the capability of NAC-SNO-nps to slowly release NO over a sustained period of time compared to the NAC-SNO solution. This slow release allows for NO to be constantly delivered to the $C$. auris planktonic cells and biofilm.

Our previous work has shown that the nanoparticle delivery platform is a viable way of delivering $\mathrm{NO}$, and delivery of these nanoparticles had substantial antibacterial and antifungal activity against both planktonic and biofilm forms [31,41,42]. This is particularly useful when considering that $C$. auris colonizes the axilla, groin, and difficult to reach areas like the ear and nose where topical administration can be effective [14]. This present study demonstrates that NAC-SNO is an effective compound for eradicating C. auris biofilms.

The spectrophotometric assay identified a dose of $10 \mathrm{mg} / \mathrm{mL}$ that was used throughout this study. This dose was also used in our past studies, as it was efficacious on bacterial species [42]. The CFU killing assays highlight the efficacy of NAC-SNO-np. NAC-SNO-np completely eradicated planktonic C. auris in all six strains. In our biofilm CFU assays, NAC-SNO-np also completely eradicated C. auris 
in the CDC strains, and resulted in growth reductions of $98 \%$ and $75 \%$ for MMC 1 and MMC 2 strains, respectively. Interestingly, it appears that there is no correlation in susceptibility of planktonic or biofilm C. auris with known resistance to antifungal drugs, a positive sign for the increasing resistance to antifungals. Moreover, C. auris grew efficiently in the 8-well chamber, and NAC-SNO-np had significant antibiofilm activity against MMC 1 and MMC 2. Confocal laser scanning microscopy corroborates this result visually as fungal cells were fewer and appeared disrupted. This indicates potent susceptibility to NAC-SNO-np. However, this decrease did not reach the $99 \%$ reduction that is considered fungicidal [43]. Despite the broad differences in susceptibilities to standard antifungals (Table 1), each C. auris strain was susceptible to the NAC-SNO-np under planktonic and biofilm growth conditions. This is a promising sign for NAC-SNO-np and NO-releasing therapeutics as a future treatment option for resistant species.

To further examine the efficacy of our nanoparticle delivery platform on C. auris biofilm, we incubated blank-np nanoparticles with $C$. auris. We have noted that our blank-np appears to have some significant effects on C. auris in our CFU killing assay for both the planktonic and biofilm forms. Our findings illustrate the capability of nanoparticles to efficiently penetrate C. auris biofilm, and we can infer from our results that the nanoparticles may inhibit biofilm growth (Figure 5). This is an interesting finding that suggests the nanoparticle scaffolding itself may inhibit the growth of $C$. auris. These effects may be due to the physico-chemical properties of the nanoparticles (e.g., particle size, charge, and surface chemistry) causing disruption in cell-to-cell communication and other cellular processes such as quorum sensing that are involved in biofilm formation [31]. We have found similar results in our previous studies with different blank particle formulations [31,41], where thiols are known to interact with disulfide bonds and react with extracellular polysaccharides, impairing ECM formation and adhesion of biofilm to surfaces $[35,36]$. In the blank-np for this experiment, the amines present on the particles may also have contributed to the antimicrobial activity of the particles [44]. While the physical presence of the blank nanoparticles may have an effect of the biofilm, the addition of NAC-SNO dramatically changes the effect of nanoparticles in the biofilm environment.

Limitations of this study include the inability to temperature regulate our $\mathrm{NO}$ analyzer to run at $37^{\circ} \mathrm{C}$, which resulted in our testing the materials at a uniform $30^{\circ} \mathrm{C}$. However, both NO and NAC-SNO release was run at the same temperature for consistency. Other limitations are the financial and time constraints related to CLSM, precluding testing on CDC strains of C. auris. However, we would expect a similar pattern of biofilm disruption from NAC-SNO-np, as demonstrated by our CFU killing assay on these strains. Other fungal strains may or may not be similarly affected by NAC-SNO-np, and future studies will need to focus on the antimicrobial effects of NAC-SNO on other fungal and bacterial species as well as investigate any cellular toxicity.

\section{Conclusions}

NAC-SNO-np effectively eradicates planktonic C. auris growth and significantly reduces and disrupts biofilm growth. NAC-SNO-np shows great promise as a future prophylactic and therapeutic for C. auris and other drug-resistant pathogens.

Author Contributions: Conceptualization, J.D.N. and J.M.F.; methodology, P.N., W.M.A., J.M.F., and J.D.N.; formal analysis, K.L.L., L.G.C., and P.N.; investigation, K.L.L., L.G.C., and P.N.; resources, P.N., J.M.F., and J.D.N.; data curation, K.L.L., L.G.C., and P.N.; writing—original draft preparation, K.L.L., L.G.C., and P.N.; writing一review and editing, W.M.A., J.M.F., and J.D.N.; visualization, K.L.L., L.G.C., and P.N.; supervision, W.M.A., J.M.F., and J.D.N. All authors have read and agreed to the published version of the manuscript.

Funding: This research received no external funding.

Acknowledgments: The authors thank Hillary Guzik and Andrea Briceno (Analytical Imaging Facility, Albert Einstein College of Medicine) for training on the use of the Leica SP5 confocal microscope and Volocity software.

Conflicts of Interest: The authors declare no conflict of interest. 


\section{References}

1. Satoh, K.; Makimura, K.; Hasumi, Y.; Nishiyama, Y.; Uchida, K.; Yamaguchi, H. Candida auris sp. nov., a novel ascomycetous yeast isolated from the external ear canal of an inpatient in a Japanese hospital. Microbiol. Immunol. 2009, 53, 41-44. [CrossRef]

2. Lockhart, S.R.; Etienne, K.A.; Vallabhaneni, S.; Farooqi, J.; Chowdhary, A.; Govender, N.P.; Colombo, A.L.; Calvo, B.; Cuomo, C.A.; Desjardins, C.A.; et al. Simultaneous Emergence of Multidrug-Resistant Candida auris on 3 Continents Confirmed by Whole-Genome Sequencing and Epidemiological Analyses. Clin. Infect. Dis. 2016, 64, 134-140. [CrossRef]

3. Kim, M.-N.; Shin, J.H.; Sung, H.; Lee, K.-W.; Kim, E.; Ryoo, N.; Lee, J.; Jung, S.I.; Park, K.H.; Kee, S.-J.; et al. Candida haemulonii and Closely Related Species at 5 University Hospitals in Korea: Identification, Antifungal Susceptibility, and Clinical Features. Clin. Infect. Dis. 2009, 48, e57-e61. [CrossRef]

4. Sarma, S.; Kumar, N.; Sharma, S.; Govil, D.; Ali, T.; Mehta, Y.; Rattan, A. Candidemia caused by amphotericin B and Fluconazole resistant Candida auris. Indian J. Med Microbiol. 2013, 31, 90. [CrossRef]

5. Magobo, R.E.; Corcoran, C.; Seetharam, S.; Govender, N.P. Candida auris-Associated Candidemia, South Africa. Emerg. Infect. Dis. 2014, 20, 1250-1252. [CrossRef]

6. Calvo, B.; Melo, A.S.D.A.; Perozo-Mena, A.; Hernandez, M.; Francisco, E.C.; Hagen, F.; Meis, J.F.; Colombo, A.L. First report of Candida auris in America: Clinical and microbiological aspects of 18 episodes of candidemia. J. Infect. 2016, 73, 369-374. [CrossRef]

7. Al-Siyabi, T.; Al Busaidi, I.; Balkhair, A.; Al-Muharrmi, Z.; Al-Salti, M.; Al'Adawi, B. First report of Candida auris in Oman: Clinical and microbiological description of five candidemia cases. J. Infect. 2017, 75, 373-376. [CrossRef]

8. Schelenz, S.; Hagen, F.; Rhodes, J.; Abdolrasouli, A.; Chowdhary, A.; Hall, A.; Ryan, L.; Shackleton, J.; Trimlett, R.; Meis, J.F.; et al. First hospital outbreak of the globally emerging Candida auris in a European hospital. Antimicrob. Resist. Infect. Control. 2016, 5, 35. [CrossRef]

9. Chow, N.A.; Gade, L.; Tsay, S.V.; Forsberg, K.; Greenko, J.A.; Southwick, K.L.; Barrett, P.M.; Kerins, J.L.; Lockhart, S.R.; Chiller, T.M.; et al. Multiple introductions and subsequent transmission of multidrug-resistant Candida auris in the USA: A molecular epidemiological survey. Lancet Infect. Dis. 2018, 18, 1377-1384. [CrossRef]

10. Chatterjee, S.; Alampalli, S.V.; Nageshan, R.K.; Chettiar, S.T.; Joshi, S.; Tatu, U. Draft genome of a commonly misdiagnosed multidrug resistant pathogen Candida auris. BMC Genom. 2015, 16, 686. [CrossRef]

11. Kathuria, S.; Singh, P.K.; Sharma, C.; Prakash, A.; Masih, A.; Kumar, A.; Meis, J.F.; Chowdhary, A. Multidrug-Resistant Candida auris Misidentified as Candida haemulonii: Characterization by Matrix-Assisted Laser Desorption Ionization-Time of Flight Mass Spectrometry and DNA Sequencing and Its Antifungal Susceptibility Profile Variability by Vitek 2, CLSI Broth Microdilution, and Etest Method. J. Clin. Microbiol. 2015, 53, 1823-1830. [CrossRef]

12. Kim, T.-H.; Kweon, O.J.; Kim, H.R.; Lee, M.-K. Identification of Uncommon Candida Species Using Commercial Identification System. J. Microbiol. Biotechnol. 2016, 26, 2206-2213. [CrossRef]

13. Horton, M.V.; Johnson, C.J.; Kernien, J.F.; Patel, T.D.; Lam, B.C.; Cheong, J.Z.A.; Meudt, J.J.; Shanmuganayagam, D.; Kalan, L.R.; Nett, J.E. Candida auris Forms High-Burden Biofilms in Skin Niche Conditions and on Porcine Skin. mSphere 2020, 5. [CrossRef]

14. Vallabhaneni, S.; Kallen, A.; Tsay, S.V.; Chow, N.; Welsh, R.; Kerins, J.; Kemble, S.K.; Pacilli, M.; Black, S.R.; Landon, E.; et al. Investigation of the First Seven Reported Cases of Candida auris, a Globally Emerging Invasive, Multidrug-Resistant Fungus-United States, May 2013-August 2016. MMWR Morb. Mortal. Wkly. Rep. 2016, 65, 1234-1237. [CrossRef]

15. Tsay, S.V.; Welsh, R.M.; Adams, E.H.; Chow, N.A.; Gade, L.; Berkow, E.L.; Poirot, E.; Lutterloh, E.; Quinn, M.; Chaturvedi, S.; et al. Notes from the Field: Ongoing Transmission of Candida auris in Health Care Facilities-United States, June 2016-May 2017. MMWR Morb. Mortal Wkly. Rep. 2017, 66, 514-515. [CrossRef]

16. Lee, W.G.; Shin, J.H.; Uh, Y.; Kang, M.G.; Kim, S.H.; Park, K.H.; Jang, H.-C. First Three Reported Cases of Nosocomial Fungemia Caused by Candida auris. J. Clin. Microbiol. 2011, 49, 3139-3142. [CrossRef]

17. Chakrabarti, A.; Sood, P.; Rudramurthy, S.M.; Chen, S.; Kaur, H.; Capoor, M.; Chhina, D.; Rao, R.; Eshwara, V.K.; Xess, I.; et al. Incidence, characteristics and outcome of ICU-acquired candidemia in India. Intensiv. Care Med. 2014, 41, 285-295. [CrossRef] 
18. Morales-López, S.E.; Parra-Giraldo, C.M.; Ceballos-Garzon, A.; Martínez, H.P.; Rodríguez, G.J.; Moreno, C.A.A.; Rodriguez, J.Y. Invasive Infections with Multidrug-Resistant Yeast Candida auris, Colombia. Emerg. Infect. Dis. 2017, 23, 162-164. [CrossRef]

19. Sears, D.; Schwartz, B.S. Candida auris: An emerging multidrug-resistant pathogen. Int. J. Infect. Dis. 2017, 63, 95-98. [CrossRef]

20. Jeffery-Smith, A.; Taori, S.K.; Schelenz, S.; Jeffery, K.; Johnson, E.M.; Borman, A.; Manuel, R.; Brown, C.S. Candida auris: A Review of the Literature. Clin. Microbiol. Rev. 2017, 31, e00029-17. [CrossRef]

21. Friedman, A.J.; Friedman, J. New biomaterials for the sustained release of nitric oxide: Past, present and future. Expert Opin. Drug Deliv. 2009, 6, 1113-1122. [CrossRef]

22. De Groote, M.A.; Fang, F.C. NO Inhibitions: Antimicrobial Properties of Nitric Oxide. Clin. Infect. Dis. 1995, 21, 162. [CrossRef]

23. Vazquez-Torres, A.; Jones-Carson, J.; Balish, E. Nitric oxide production does not directly increase macrophage candidacidal activity. Infect. Immun. 1995, 63, 1142-1144. [CrossRef]

24. Jones-Carson, J.; Vázquez-Torres, A.; Van Der Heyde, H.C.; Warner, T.; Wagner, R.D.; Balish, E. $\gamma \delta \mathrm{T}$ cell-induced nitric oxide production enhances resistance to mucosal candidiasis. Nat. Med. 1995, 1, 552-557. [CrossRef]

25. Ahmadi, M.S.; Lee, H.H.; Sanchez, D.A.; Friedman, A.J.; Tar, M.T.; Davies, K.E.; Nosanchuk, J.D.; Martinez, L.R. Sustained Nitric Oxide-Releasing Nanoparticles Induce Cell Death in Candida albicans Yeast and Hyphal Cells, Preventing Biofilm Formation In Vitro and in a Rodent Central Venous Catheter Model. Antimicrob. Agents Chemother. 2016, 60, 2185-2194. [CrossRef]

26. Han, G.; Martinez, L.R.; Mihu, M.R.; Friedman, A.J.; Friedman, J.M.; Nosanchuk, J.D. Nitric Oxide Releasing Nanoparticles Are Therapeutic for Staphylococcus aureus Abscesses in a Murine Model of Infection. PLoS ONE 2009, 4, e7804. [CrossRef]

27. Martinez, L.R.; Han, G.; Chacko, M.; Mihu, M.R.; Jacobson, M.; Gialanella, P.; Friedman, A.J.; Nosanchuk, J.D.; Friedman, J.M. Antimicrobial and Healing Efficacy of Sustained Release Nitric Oxide Nanoparticles Against Staphylococcus Aureus Skin Infection. J. Investig. Dermatol. 2009, 129, 2463-2469. [CrossRef]

28. Vargas-Cruz, N.; Reitzel, R.A.; Rosenblatt, J.; Chaftari, A.-M.; Dib, R.W.; Hachem, R.; Kontoyiannis, D.P.; Raad, I.I. Nitroglycerin-Citrate-Ethanol Catheter Lock Solution Is Highly Effective for In Vitro Eradication of Candida auris Biofilm. Antimicrob. Agents Chemother. 2019, 63, 63. [CrossRef]

29. Zamith-Miranda, D.; Heyman, H.M.; Cleare, L.G.; Couvillion, S.P.; Clair, G.; Bredeweg, E.L.; Gacser, A.; Nimrichter, L.; Nakayasu, E.S.; Nosanchuk, J.D. Multi-omics Signature of Candida auris, an Emerging and Multidrug-Resistant Pathogen. mSystems 2019, 4, e00257-19. [CrossRef]

30. Brinker, C.; Keefer, K.; Schaefer, D.; Ashley, C. Sol-gel transition in simple silicates. J. Non-Cryst. Solids 1982, 48, 47-64. [CrossRef]

31. Girish, V.M.; Liang, H.; Aguilan, J.T.; Nosanchuk, J.D.; Friedman, J.M.; Nacharaju, P. Anti-biofilm activity of garlic extract loaded nanoparticles. Nanomed. Nanotechnol. Biol. Med. 2019, 20, 102009. [CrossRef]

32. De Oliveira, M.G.; Shishido, S.M.; Seabra, A.B.; Morgon, N. Thermal Stability of Primary S-Nitrosothiols: Roles of Autocatalysis and Structural Effects on the Rate of Nitric Oxide Release. J. Phys. Chem. A 2002, 106, 8963-8970. [CrossRef]

33. Fanning, S.; Mitchell, A.P. Fungal Biofilms. PLoS Pathog. 2012, 8, e1002585. [CrossRef]

34. Kean, R.; Delaney, C.; Sherry, L.; Borman, A.; Johnson, E.M.; Richardson, M.D.; Richardson, R.R.; Williams, C.; Ramage, G. Transcriptome Assembly and Profiling of Candida auris Reveals Novel Insights into Biofilm-Mediated Resistance. mSphere 2018, 3, e00334-18. [CrossRef]

35. Moon, J.-H.; Choi, Y.-S.; Lee, H.-W.; Heo, J.S.; Chang, S.W.; Lee, J.-Y. Antibacterial effects of N-acetylcysteine against endodontic pathogens. J. Microbiol. 2016, 54, 322-329. [CrossRef]

36. Kręgiel, D.; Rygala, A.; Kolesinska, B.; Nowacka, M.; Herc, A.S.; Kowalewska, A. Antimicrobial and Antibiofilm N-acetyl-L-cysteine Grafted Siloxane Polymers with Potential for Use in Water Systems. Int. J. Mol. Sci. 2019, 20, 2011. [CrossRef]

37. Blasi, F.; Page, C.P.; Rossolini, G.; Pallecchi, L.; Matera, M.G.; Rogliani, P.; Cazzola, M. The effect of N -acetylcysteine on biofilms: Implications for the treatment of respiratory tract infections. Respir. Med. 2016, 117, 190-197. [CrossRef] 
38. Fernández-Rivero, M.E.; Del Pozo, J.; Valentín, A.; De Diego, A.M.; Pemán, J.; Cantón, E. Activity of Amphotericin B and Anidulafungin Combined with Rifampicin, Clarithromycin, Ethylenediaminetetraacetic Acid, N-Acetylcysteine, and Farnesol against Candida tropicalis Biofilms. J. Fungi 2017, 3, 16. [CrossRef]

39. Ercan, U.K.; Smith, J.; Ji, H.-F.; Brooks, A.D.; Joshi, S.G. Chemical Changes in Nonthermal Plasma-Treated N-Acetylcysteine (NAC) Solution and Their Contribution to Bacterial Inactivation. Sci. Rep. 2016, 6, 20365. [CrossRef]

40. Sehajpal, P.K.; Basu, A.; Ogiste, J.S.; Lander, H.M. Reversible S-Nitrosation and Inhibition of HIV-1 Protease. Biochemistry 1999, 38, 13407-13413. [CrossRef]

41. Ba, C.Y.; Mavelli, G.V.; Nacharaju, P.; Li, K.; Cleare, L.G.; Nosanchuk, J.D.; Friedman, J.M.; Abuzeid, W.M. Novel nitric oxide-generating platform using manuka honey as an anti-biofilm strategy in chronic rhinosinusitis. Int. Forum Allergy Rhinol. 2019, 10, 223-232. [CrossRef]

42. Abuzeid, W.M.; Girish, V.M.; Fastenberg, J.H.; Draganski, A.R.; Lee, A.Y.; Nosanchuk, J.D.; Friedman, J.M. Nitric oxide-releasing microparticles as a potent antimicrobial therapeutic against chronic rhinosinusitis bacterial isolates. Int. Forum Allergy Rhinol. 2018, 8, 1190-1198. [CrossRef]

43. Graybill, J.R.; Burgess, D.S.; Hardin, T.C. Key issues concerning fungistatic versus fungicidal drugs. Eur. J. Clin. Microbiol. Infect. Dis. 1997, 16, 42-50. [CrossRef]

44. Endo, Y.; Tani, T.; Kodama, M. Antimicrobial activity of tertiary amine covalently bonded to a polystyrene fiber. Appl. Environ. Microbiol. 1987, 53, 2050-2055. [CrossRef]

(C) 2020 by the authors. Licensee MDPI, Basel, Switzerland. This article is an open access article distributed under the terms and conditions of the Creative Commons Attribution (CC BY) license (http://creativecommons.org/licenses/by/4.0/). 